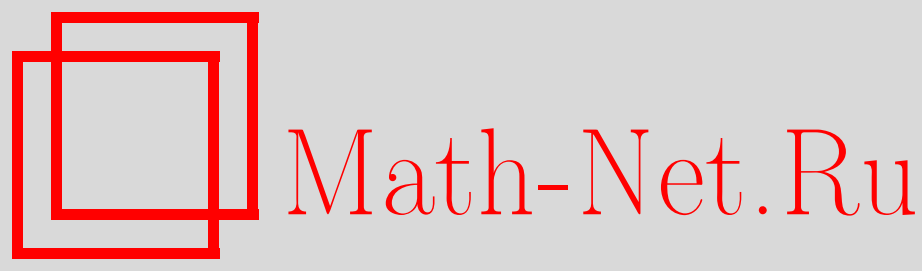

А. С. Овчинников, И. Г. Бострем, В. Е. Синицын, Д. Кишине, Критическое поведение моноаксиального кирального гелимагнетика, ТMФ, 2017, том 191, номер 3, 518534

DOI: https://doi.org/10.4213/tmf9223

Использование Общероссийского математического портала Math-Net.Ru подразумевает, что вы прочитали и согласны с пользовательским соглашением http://www.mathnet.ru/rus/agreement

Параметры загрузки:

IP: 3.85 .73 .92

26 апреля 2023 г., 14:31:07

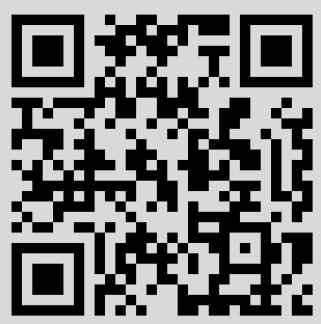




\title{
КРИТИЧЕСКОЕ ПОВЕДЕНИЕ МОНОАКСИАЛЬНОГО КИРАЛЬНОГО ГЕЛИМАГНЕТИКА
}

\begin{abstract}
Анализируется критическое поведение магнитоупорядоченных фаз в моноаксиальном киральном гелимагнетике, возникающих в слабом внешнем магнитном поле. С помощью формализма уравнений состояния в критической области определяется температурная зависимость параметров порядка для конической фазы и фазы солитонной решетки. Выполнен расчет критических показателей, показано, что они совпадают со значениями показателей в трехмерной модели Гейзенберга.
\end{abstract}

Ключевые слова: взаимодействие Дзялошинского-Мории, фазовые переходы, критические индексы.

DOI: https://doi.org/10.4213/tmf9223

\section{1. ВВЕДЕНИЕ}

Магнитные кристаллы, принадлежащие киральным пространственным группам с отсутствием центра инверсии, такие как $\mathrm{MnSi}$ [1], [2], $\mathrm{Fe}_{1-x} \mathrm{Co}_{x} \mathrm{Si}$ [3], [4], FeGe [5], [6], $\mathrm{Cu}_{2} \mathrm{OSeO}_{5}$ [7], [8] и $\mathrm{CrNb}_{3} \mathrm{~S}_{6}$ [9], [10], привлекают повышенное внимание из-за разнообразия наблюдаемых нетривиальных магнитных фаз, из которых наиболее интересны скирмионная фаза [2], [11], [12] и фаза солитонной решетки [13]-[16]. Считается, что взаимодействие Дзялошинского-Мории (ДМ) [17], [18] играет решающую роль в стабилизации этих пространственно-неоднородных структур.

Работа поддержана Japan Society for the Promotion of Science, KAKENHI Proramm (гранты 25287087, 25220803), а также Правительством РФ, акт 211 (договор № 02.А03.21.0006) и Министерством образования и науки РФ (проекты № 1437 и № 2725). Вл. Е. Синицын благодарит за поддержку Программу поддержки молодых ученых - кандидатов наук (грант № MK-6230.2016.2).

* Институт естественных наук, Уральский федеральный университет им. первого Президента России Б. Н. Ельцина, Екатеринбург, Россия. E-mail: alexander.ovchinnikov@urfu.ru

${ }^{\dagger}$ Division of Natural and Environmental Sciences, The Open University of Japan, Chiba, Japan 
Магнитная фазовая диаграмма нецентросимметричных материалов зависит от кристаллографической пространственной группы. В частности, в случае гексагональных, тетрагональных и тригональных киральных кристаллов имеется моноаксиальное взаимодействие ДМ вдоль главной (киральной) оси, которое стабилизирует гелимагнитный порядок. Свойства основного состояния гелимагнитной структуры во внешнем магнитном поле, приложенном параллельно или перпендикулярно киральной оси, достаточно хорошо изучены [13], [14], [16]. Под действием параллельного поля с его увеличением основное состояние сначала изменяется от плоской спирали к конической, а затем переходит в состояние вынужденного ферромагнетизма. Под действием возрастающего перпендикулярного поля основное состояние эволюционирует от плоской спирали к состоянию солитонной решетки; этот процесс намагничивания также завершется фазой вынужденного ферромагнетизма. Данная последовательность фазовых переходов наблюдалась экспериментально в слоистом гексагональном киральном гелимагнетике $\mathrm{CrNb}_{3} \mathrm{~S}_{6}$ [15], [19], [20].

Гелимагнитная структура также встречается в кубических магнетиках, например в соединениях В20 с пространственной группой Р2 $2_{1} 3$. В последнем случае слабая кубическая анизотропия определяет направление магнитной спирали вдоль главных осей кубической симметрии. При наложении магнитного поля волновой вектор спирали поворачивается, выравниваясь вдоль направления поля, начиная с определенного значения, которое зависит от величины кубической анизотропии. Одновременно спираль трансформируется в коническую структуру с волновым вектором, направленным вдоль оси поля [21], [22]. Этот порядок остается стабильным вплоть до второго критического значения поля, когда все спины становятся ориентированы ферромагнитным образом.

В сравнении со свойствами основного состояния гораздо меньше внимания было уделено температурным фазовым переходам и критическому поведению в фазовой плоскости “температура-магнитное поле". В случае кубических киральных гелимагнетиков основными проблемами являются объяснение происхождения так называемой А-фазы и природа фазового перехода первого рода из парамагнитной в гелимагнитную фазу в нулевом магнитном поле в металлическом MnSi [23]. Для того чтобы объяснить это необычное поведение, была использована теория Бразовского [24], которая предсказывает, что из-за взаимодействия ДМ система не претерпевает фазовый переход второго рода, и это обстоятельство лежит в основе объяснения явления скирмионной жидкости в узком интервале фазовой диаграммы выше

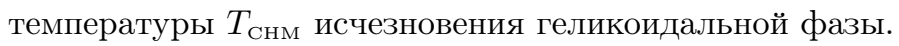

Но даже в случае гелимагнетика $\mathrm{Cr}_{1 / 3} \mathrm{NbS}_{2}$ с гексагональной симметрией детали того, как возникает солитонная решетка из парамагнитной фазы при охлаждении, остаются до конца невыясненными. Во-первых отметим, что имеется существенный разброс значений критической температуры в нулевом магнитном поле [19], [25], [26], что может быть связано с качеством исследуемых образцов [26]. Вторая особенность для случая $\mathrm{Cr}_{1 / 3} \mathrm{NbS}_{2}$ состоит в наличии $\lambda$-образной сингулярности в кривой намагничивания в малом магнитном поле (меньше чем $1 \mathrm{kOe}$ ), приложенном перпендикулярно с-оси. Эта особенность отсутствует в более высоких полях, что можно рассматривать как возникновение геликоидального порядка. Об обнаружении этой сингулярности было впервые сообщено в работе [10] и подтверждено в недавних экспериментах [19], [20], выполненых на монокристаллах больших образцов. При 
температурах выше этой особенности наблюдается поведение Кюри-Вейсса и система претерпевает кроссовер в парамагнитную фазу [20]. Из анализа экспериментальных данных было найдено, что кроссовер принадлежит классу универсальности трехмерной модели Гейзенберга [26]. Отметим, что подобный вывод был сделан для кубического гелимагнетика $\mathrm{Cu}_{2} \mathrm{OSeO}_{3}$ [27]. Это же подтверждается недавним численным исследованием трехмерной классической спиновой модели кирального гелимагнетика методом Монте-Карло [28]. В частности, было показано, что в нулевом магнитном поле система испытывает непрерывный фазовый переход с критическими индексами трехмерной ферромагнитной $X Y$-модели. Эти экспериментальные и численные данные показывают, что взаимодействие ДМ не вносит каких-либо дополнительных кроссоверных индексов.

В настоящей статье мы показываем, что сингулярную особенность можно объяснить тем, что несоизмеримый волновой вектор зависит от температуры, и интерпретировать как фазовый переход соизмеримая фаза-несоизмеримая фаза. Экспериментальный результат, который свидетельствует о том, что $\mathrm{Cr}_{1 / 3} \mathrm{NbS}_{2}$ обладает классом универсальности трехмерной модели Гейзенберга, согласуется с ренормгрупповым анализом модели кирального гелимагнетика в парамагнитной фазе [29], но противоречит ранним исследованиям этого вопроса [30]. Тем не менее, насколько нам известно, теоретический анализ критического поведения со стороны упорядоченных фаз отсутствует. В статье мы проводим исследование проблемы, основываясь на формализме уравнения состояния в критической области, разработанном в работе [31]. Особое внимание уделено расчету критических показателей; показано, что они совпадают со значениями критических показателей в трехмерной модели Гейзенберга [32].

Статья организована следующим образом. В разделе 2 дано описание модели. В п. 3.1 описывается общая схема формализма уравнения состояния магнитоупорядоченных фаз в критической области. Критическое поведение конической фазы в случае слабого внешнего магнитного поля исследовано в п. 3.2. Переход от солитонной решетки к ферромагнитному состоянию и кроссовер из фазы вынужденного ферромагнетизма в парамагнитную фазу исследованы в п. 3.3 и п. 3.4 соответственно. Обобщение результатов представлено в разделе 4.

\section{2. МОДЕЛЬ}

Гамильтониан слоистого кирального гелимагнетика, прототипа реального соединения $\mathrm{CrNb}_{3} \mathrm{~S}_{6}$, с двумя типами обменных взаимодействий (см. рис. 1), а именно внутрислойным взаимодействием $J_{\perp}$ и межслойным взаимодействием $J_{z}$, имеет вид

$$
\mathcal{H}=\mathcal{H}_{\text {ex }}+\mathcal{H}_{\text {an }}+\mathcal{H}_{\mathrm{DM}}
$$

Первое слагаемое описывает обменное взаимодействие между парами ближайших соседей $\langle i j\rangle$ внутри плоскости и вдоль геликоидальной оси:

$$
\mathcal{H}_{\mathrm{ex}}=-\frac{J_{\perp}}{2} \sum_{\langle i j\rangle} \mathbf{S}_{i} \cdot \mathbf{S}_{j}-\frac{J_{z}}{2} \sum_{\langle i j\rangle} \mathbf{S}_{i} \cdot \mathbf{S}_{j}
$$




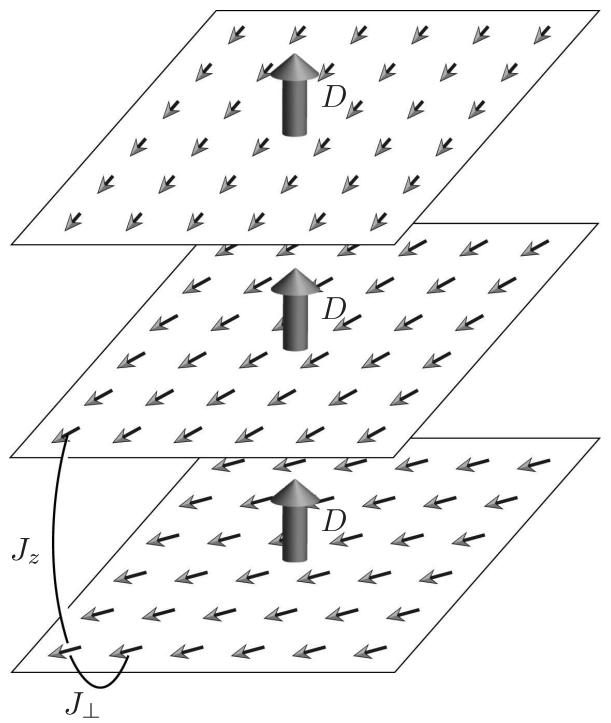

Рис. 1. Модель кирального моноаксиального гелимагнетика.

где $\mathbf{S}_{i}$ - трехкомпонентный вектор классического спина, расположенного на узле $i$. Второе слагаемое соответствует одноосной одноионной анизотропии:

$$
\mathcal{H}_{\mathrm{an}}=\frac{g}{2} \sum_{i}\left[S_{i}^{z} S_{i}^{z}-\frac{1}{2}\left(S_{i}^{x} S_{i}^{x}+S_{i}^{y} S_{i}^{y}\right)\right] .
$$

Третье слагаемое - взаимодействие ДМ с константой $D$,

$$
\mathcal{H}_{\mathrm{DM}}=D \sum_{\langle i j\rangle^{\prime}}\left(S_{i}^{x} S_{j}^{y}-S_{j}^{x} S_{i}^{y}\right),
$$

в котором $\langle i j\rangle^{\prime}$ соответствует ближайшим соседям вдоль оси $z$; предполагается, что узел $j$ расположен в положительном направлении по отношению к узлу $i$.

С помощью фурье-преобразования

$$
S^{\alpha}(\mathbf{r})=\frac{1}{\sqrt{V}} \sum_{\mathbf{k}} e^{i \mathbf{k r}} S_{\mathbf{k}}^{\alpha}
$$

преобразуем гамильтониан (1) к виду

$$
\begin{aligned}
\mathcal{H}= & \frac{1}{2} \sum_{\mathbf{k}}\left\{\left(J_{\perp} \mathbf{k}^{2}+J_{z} k_{z}^{2}\right)\left(\mathbf{S}_{\mathbf{k}} \cdot \mathbf{S}_{-\mathbf{k}}\right)+g\left[S_{\mathbf{k}}^{z} S_{-\mathbf{k}}^{z}-\frac{1}{2}\left(S_{\mathbf{k}}^{x} S_{-\mathbf{k}}^{x}+S_{\mathbf{k}}^{y} S_{-\mathbf{k}}^{y}\right)\right]\right\}- \\
& -2 i D \sum_{\mathbf{k}} k_{z} S_{\mathbf{k}}^{x} S_{-\mathbf{k}}^{y} .
\end{aligned}
$$

Диагонализация гамильтониана приводит к квадратичной форме [29]

$$
\mathcal{H}=\frac{1}{2} \sum_{\mathbf{k}} \sum_{\alpha=x, y, x}\left(r_{\alpha}+\mathbf{k}_{\perp}^{2}+\Delta \mathbf{k}_{z}^{2}\right) \sigma_{\mathbf{k}}^{\alpha} \sigma_{-\mathbf{k}}^{\alpha},
$$


в которой $\Delta=J_{z} / J_{\perp}, r_{x}=r_{y}=\rho-g / 2-D^{2} / J_{z}, r_{z}=\rho+g$; операторы $\sigma_{\mathbf{k}}^{ \pm}$определяются стандартным образом как операторы повышения/понижения:

$$
\sigma_{\mathbf{k}}^{ \pm}=\frac{1}{\sqrt{2}}\left(S_{\mathbf{k} \mp Q_{0} \mathbf{e}_{z}}^{x} \pm i S_{\mathbf{k} \mp Q_{0} \mathbf{e}_{z}}^{y}\right), \quad \sigma_{\mathbf{k}}^{z}=S_{\mathbf{k}}^{z},
$$

где $\mathbf{Q}_{0}=\left(D / J_{z}\right) \mathbf{e}_{z}$. В дальнейшем предполагается, что $J_{\perp}=1$ и $\rho=a\left(T_{0}-T\right)$, где $T_{0}$ - температура упорядочения в теории среднего поля для изотропной системы Гейзенберга, $a$ - положительная константа.

В моноаксиальном киральном гелимагнетике под действием внешнего поля, приложенного вдоль геликоидальной оси или перпендикулярно ей, возникают соответственно коническая фаза или фаза солитонной решетки. Для учета зеемановского взаимодействия удобно перейти от исходных спиновых переменных $\mathbf{S}_{\mathbf{k}}^{\alpha}$ к новым величинам $\sigma_{\mathbf{k}}^{\alpha}$ :

$$
\mathcal{H}_{\mathrm{Z}}=\sum_{\mathbf{k}, \alpha} h_{\mathbf{k}}^{\alpha} S_{-\mathbf{k}}^{\alpha}=\sum_{\mathbf{k}, \alpha} \tilde{h}_{\mathbf{k}}^{\alpha} \sigma_{-\mathbf{k}}^{\alpha}
$$

где компоненты эффективного магнитного поля, действующего на новые спиновые переменные, определены как

$$
\begin{aligned}
& \tilde{h}_{\mathbf{k}}^{x}=\frac{1}{2}\left(h_{\mathbf{k}+\mathbf{Q}_{0}}^{x}-i h_{\mathbf{k}+\mathbf{Q}_{0}}^{y}+h_{\mathbf{k}-\mathbf{Q}_{0}}^{x}+i h_{\mathbf{k}-\mathbf{Q}_{0}}^{y}\right), \\
& \tilde{h}_{\mathbf{k}}^{y}=\frac{i}{2}\left(h_{\mathbf{k}+\mathbf{Q}_{0}}^{x}-i h_{\mathbf{k}+\mathbf{Q}_{0}}^{y}-h_{\mathbf{k}-\mathbf{Q}_{0}}^{x}-i h_{\mathbf{k}-\mathbf{Q}_{0}}^{y}\right), \\
& \tilde{h}_{\mathbf{k}}^{z}=h_{\mathbf{k}}^{z} .
\end{aligned}
$$

\section{3. УРАВНЕНИЕ СОСТОЯНИЯ}

3.1. Теория возмущений. Для математического вывода уравнений состояний определим флуктуации новых полей

$$
\sigma_{\mathbf{k}}^{\alpha}=\sigma_{\mathbf{k}}^{\alpha}-m_{\mathbf{k}}^{\alpha},
$$

в которых пространственно неоднородная фаза описывается векторным параметром порядка

$$
m^{\alpha}(\mathbf{r})=\frac{1}{\sqrt{V}} \sum_{\mathbf{k}} e^{i \mathbf{k r}} m_{\mathbf{k}}^{\alpha}
$$

и принято условие, что $\left\langle\sigma_{\mathbf{k}}^{\prime \alpha}\right\rangle=0$.

С учетом флуктуаций исходный гамильтониан приобретает вид

$$
\begin{aligned}
\mathcal{H}_{0}\left[\mathbf{m}, \boldsymbol{\sigma}^{\prime}\right]=\frac{1}{2} \sum_{\mathbf{k}, \alpha}\left[\left(r_{\alpha}\right.\right. & \left.+\mathbf{k}_{\perp}^{2}+\Delta \mathbf{k}_{z}^{2}\right) \sigma_{\mathbf{k}}^{\prime \alpha} \sigma_{-\mathbf{k}}^{\prime \alpha}+2\left(r_{\alpha}+\mathbf{k}_{\perp}^{2}+\Delta \mathbf{k}_{z}^{2}\right) m_{-\mathbf{k}}^{\alpha} \sigma_{\mathbf{k}}^{\prime \alpha}+ \\
& \left.+\left(r_{\alpha}+\mathbf{k}_{\perp}^{2}+\Delta \mathbf{k}_{z}^{2}\right) m_{\mathbf{k}}^{\alpha} m_{-\mathbf{k}}^{\alpha}\right]-\sum_{\mathbf{k}, \alpha} \tilde{h}_{\mathbf{k}}^{\alpha}\left(m_{-\mathbf{k}}^{\alpha}+\sigma_{-\mathbf{k}}^{\prime \alpha}\right) .
\end{aligned}
$$


Рис. 2. Вклад нулевого порядка в среднее $\left\langle\boldsymbol{\sigma}^{\prime}\right\rangle$.

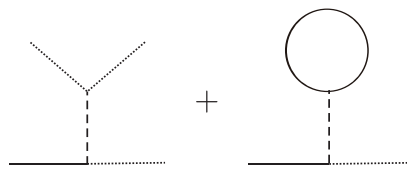

Рис. 3. Вклады первого порядка в среднее $\left\langle\boldsymbol{\sigma}^{\prime}\right\rangle$.

Для дополнительного описания взаимодействия флуктуаций выбирается гамильтониан, форма которого тождественна той, которая используется в стандартной $\phi^{4}$-теории:

$$
\begin{aligned}
\mathcal{H}_{\mathrm{int}}=\frac{u}{8} \int d \mathbf{r} \sigma^{4}(\mathbf{r}) & = \\
=\frac{u}{8} \sum_{\mathbf{k}_{1}, \mathbf{k}_{2}, \mathbf{k}_{3}, \mathbf{k}_{4}}\{ & {\left[\left(\mathbf{m}_{\mathbf{k}_{1}} \cdot \mathbf{m}_{\mathbf{k}_{2}}\right)\left(\mathbf{m}_{\mathbf{k}_{3}} \cdot \mathbf{m}_{\mathbf{k}_{4}}\right)+\left(\boldsymbol{\sigma}_{\mathbf{k}_{1}}^{\prime} \cdot \boldsymbol{\sigma}_{\mathbf{k}_{2}}^{\prime}\right)\left(\boldsymbol{\sigma}_{\mathbf{k}_{3}}^{\prime} \cdot \boldsymbol{\sigma}_{\mathbf{k}_{4}}^{\prime}\right)\right]+} \\
+ & 4\left[\left(\mathbf{m}_{\mathbf{k}_{1}} \cdot \mathbf{m}_{\mathbf{k}_{2}}\right)\left(\mathbf{m}_{\mathbf{k}_{3}} \cdot \boldsymbol{\sigma}_{\mathbf{k}_{4}}^{\prime}\right)+\left(\mathbf{m}_{\mathbf{k}_{1}} \cdot \boldsymbol{\sigma}_{\mathbf{k}_{2}}^{\prime}\right)\left(\boldsymbol{\sigma}_{\mathbf{k}_{3}}^{\prime} \cdot \boldsymbol{\sigma}_{\mathbf{k}_{4}}^{\prime}\right)\right]+ \\
& \left.+2\left[\left(\mathbf{m}_{\mathbf{k}_{1}} \cdot \mathbf{m}_{\mathbf{k}_{2}}\right)\left(\boldsymbol{\sigma}_{\mathbf{k}_{3}}^{\prime} \cdot \boldsymbol{\sigma}_{\mathbf{k}_{4}}^{\prime}\right)+2\left(\mathbf{m}_{\mathbf{k}_{1}} \cdot \boldsymbol{\sigma}_{\mathbf{k}_{2}}^{\prime}\right)\left(\mathbf{m}_{\mathbf{k}_{3}} \cdot \boldsymbol{\sigma}_{\mathbf{k}_{4}}^{\prime}\right)\right]\right\} \times \\
& \times \delta\left(\mathbf{k}_{1}+\mathbf{k}_{2}+\mathbf{k}_{3}+\mathbf{k}_{4}\right) .
\end{aligned}
$$

Уравнение состояния получается непосредственно из условия $\left\langle\boldsymbol{\sigma}_{\mathbf{k}}^{\prime \alpha}\right\rangle=0$. Здесь термодинамическое среднее определено как функциональный интеграл:

$$
\langle\cdot\rangle \equiv \frac{\int \mathcal{D} \sigma^{\prime} e^{-\mathcal{H}}(\cdot)}{\int \mathcal{D} \sigma^{\prime} e^{-\mathcal{H}}},
$$

где $\mathcal{H}=\mathcal{H}_{0}+\mathcal{H}_{\text {int }}$ и для его вычисления используется теория возмущений [31].

Слагаемое нулевого порядка определяется диаграммой типа "головастик" (см. рис. 2), в которой круг соответствует эффективному полю $\tilde{h}_{\mathbf{k}}^{\alpha}-m_{\mathbf{k}}^{\alpha}\left[r_{\alpha}+\mathbf{k}_{\perp}^{2}+\Delta \mathbf{k}_{z}^{2}\right]$, а сплошная линия обозначает функцию Грина

$$
G\left(\mathbf{k}, r_{\alpha}\right)=\frac{1}{r_{\alpha}+\mathbf{k}_{\perp}^{2}+\Delta \mathbf{k}_{z}^{2}} .
$$

Вклады первого порядка вычисляются с помощью диаграмм, показанных на рис. 3, в которых пунктирные линии соответствуют $m_{\mathbf{k}}^{\alpha}$, а штриховые линии обозначают взаимодействие $u$.

В конечном итоге термодинамическое уравнение состояния приобретает форму

$$
\begin{aligned}
\tilde{h}_{\mathbf{k}}^{\alpha}= & m_{\mathbf{k}}^{\alpha}\left(r_{\alpha}+\mathbf{k}_{\perp}^{2}+\Delta \mathbf{k}_{z}^{2}\right)+\frac{u}{2} \sum_{\mathbf{k}_{1}, \mathbf{k}_{2}} m_{\mathbf{k}-\mathbf{k}_{1}-\mathbf{k}_{2}}^{\alpha}\left(\mathbf{m}_{\mathbf{k}_{1}} \cdot \mathbf{m}_{\mathbf{k}_{2}}\right)+ \\
& +\frac{u}{2} m_{\mathbf{k}}^{\alpha} \sum_{\mathbf{p}, \beta} G\left(\mathbf{p}, r_{\beta}\right)+u m_{\mathbf{k}}^{\alpha} \sum_{\mathbf{p}} G\left(\mathbf{p}, r_{\alpha}\right) .
\end{aligned}
$$

В приближении Хартри-Фока условие самосогласования достигается путем наложения требования, что уравнение состояния должно определяться функциями Грина $G_{\alpha}(\mathbf{p})=\left\langle\sigma_{\mathbf{p}}^{\prime \alpha} \sigma_{-\mathbf{p}}^{\prime \alpha}\right\rangle$, которые имеют вид $(13)$, но зависят от перенормированной 


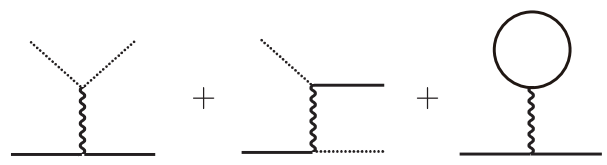

Рис. 4. Вклады первого порядка в собственно-энергетическую часть $\Sigma$.

константы взаимодействия $\tilde{r}_{\alpha}$ вместо затравочного значения $r_{\alpha}$ [24], [32]:

$$
\begin{aligned}
\tilde{h}_{\mathbf{k}}^{\alpha}= & m_{\mathbf{k}}^{\alpha}\left(r_{\alpha}+\mathbf{k}_{\perp}^{2}+\Delta \mathbf{k}_{z}^{2}\right)+\frac{u}{2} \sum_{\mathbf{k}_{1}, \mathbf{k}_{2}} m_{\mathbf{k}-\mathbf{k}_{1}-\mathbf{k}_{2}}^{\alpha}\left(\mathbf{m}_{\mathbf{k}_{1}} \cdot \mathbf{m}_{\mathbf{k}_{2}}\right)+ \\
& +\frac{u}{2} m_{\mathbf{k}}^{\alpha} \sum_{\mathbf{p}, \beta} G\left(\mathbf{p}, \tilde{r}_{\beta}\right)+u m_{\mathbf{k}}^{\alpha} \sum_{\mathbf{p}} G\left(\mathbf{p}, \tilde{r}_{\alpha}\right) .
\end{aligned}
$$

Из уравнения Дайсона $G_{\alpha}^{-1}=G_{\alpha}^{(0)^{-1}}+\Sigma_{\alpha}$ следует, что $\tilde{r}_{\alpha}=r_{\alpha}+\Sigma_{\alpha}$. Собственноэнергетическая часть $\Sigma_{\alpha}$ определяется диаграммами, изображенными на рис. 4 , что приводит к результату

$$
\tilde{r}_{\alpha}=r_{\alpha}+\frac{u}{2} \sum_{\mathbf{k}}\left(\mathbf{m}_{\mathbf{k}} \cdot \mathbf{m}_{-\mathbf{k}}\right)+u \sum_{\mathbf{k}} m_{\mathbf{k}}^{\alpha} m_{-\mathbf{k}}^{\alpha}+u \sum_{\mathbf{p}} G\left(\mathbf{p}, \tilde{r}_{\alpha}\right)+\frac{u}{2} \sum_{\mathbf{p}, \beta} G\left(\mathbf{p}, \tilde{r}_{\beta}\right) .
$$

Уравнения (15), (16) образуют замкнутую систему, лежащую в основе анализа критического поведения различных фаз моноаксиального кирального гелимагнетика.

3.2. Коническая фаза. Коническая фаза возникает под действием внешнего магнитного поля, приложенного вдоль геликоидальной оси перпендикулярно слоям. В отсутствии поля основное состояние системы представляет собой планарное ферромагнитное упорядочение спинов с ненулевым средним значением $\langle\mathbf{S}\rangle$ вдоль некоторого направления в $x y$-плоскости: $\left\langle S^{x}\right\rangle+i\left\langle S^{y}\right\rangle=m_{\perp} e^{i \varphi}$ и $\left\langle S^{z}\right\rangle=0$, где $m_{\perp}$ положительно и угол $\varphi$ определяется значением несоизмеримости $Q_{0}$. Планарное упорядочение спинов реализуется при условии, что $J_{\perp}$ больше $J_{z}$.

Под действием внешнего поля $h$, меньшего некоторого критического значения, возникает коническая фаза, основное состояние в которой обладает плоскостным ферромагнитным упорядочением с $m_{\perp} \neq 0$, но при этом одновременно становится ненулевым и упорядочение вдоль геликоидальной оси, $\left\langle S^{z}\right\rangle=m_{z}$. Для данной фазы параметризация спинов имеет вид $S_{x}=m_{\perp} \cos Q_{0} z, S_{y}=m_{\perp} \sin Q_{0} z$ и $S_{z}=m_{z}$ с условием $m_{\perp}^{2}+m_{z}^{2}=m^{2}$.

Ненулевые гармоники фурье-разложения

$$
S_{\mathbf{Q}_{0}}^{x}=S_{-\mathbf{Q}_{0}}^{x}=\frac{m_{\perp}}{2}, \quad S_{\mathbf{Q}_{0}}^{y}=\left(S_{-\mathbf{Q}_{0}}^{y}\right)^{*}=i \frac{m_{\perp}}{2}
$$

определяют необходимые компоненты фоновой намагниченности

$$
m_{\mathbf{k}}^{x}=m_{\perp} \delta_{\mathbf{k}, \mathbf{0}}, \quad m_{\mathbf{k}}^{y}=0, \quad m_{\mathbf{k}}^{z}=m_{z} \delta_{\mathbf{k}, \mathbf{0}}
$$

и магнитного поля $\tilde{h}_{\mathbf{k}}^{\alpha}=h \delta_{\alpha, z} \delta_{\mathbf{k}, \mathbf{0}}$. 


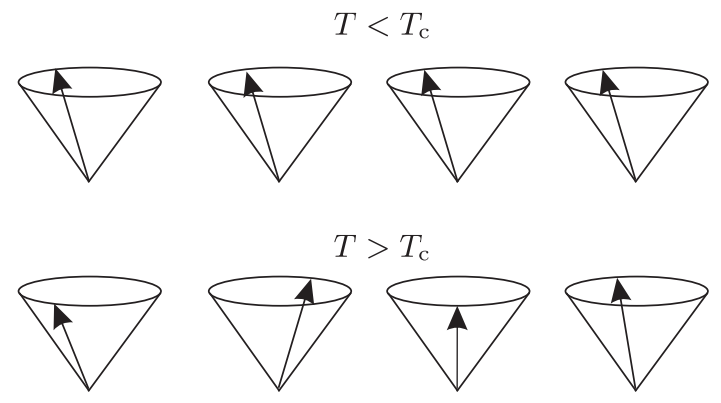

Рис. 5. Фазовый переход в конической фазе.

Уравнения (15) и (16) принимают вид

$$
\begin{aligned}
\frac{h}{m_{z}} & =r_{z}+\frac{u}{2} m^{2}+\frac{u}{2}(n-1) \sum_{\mathbf{p}} G\left(\mathbf{p}, \tilde{r}_{\perp}\right)+\frac{3}{2} u \sum_{\mathbf{p}} G\left(\mathbf{p}, \tilde{r}_{z}\right), \\
0 & =r_{x}+\frac{u}{2} m^{2}+\frac{u}{2}(n+1) \sum_{\mathbf{p}} G\left(\mathbf{p}, \tilde{r}_{\perp}\right)+\frac{u}{2} \sum_{\mathbf{p}} G\left(\mathbf{p}, \tilde{r}_{z}\right),
\end{aligned}
$$

где $n=3$ - число спиновых компонент и

$$
\begin{aligned}
& \tilde{r}_{\perp}=r_{x}+\frac{u}{2} m^{2}+u m_{\perp}^{2}+\frac{u}{2}(n+1) \sum_{\mathbf{p}} G\left(\mathbf{p}, \tilde{r}_{\perp}\right)+\frac{u}{2} \sum_{\mathbf{p}} G\left(\mathbf{p}, \tilde{r}_{z}\right), \\
& \tilde{r}_{z}=r_{z}+\frac{u}{2} m^{2}+u m_{z}^{2}+\frac{u}{2}(n-1) \sum_{\mathbf{p}} G\left(\mathbf{p}, \tilde{r}_{\perp}\right)+\frac{3 u}{2} \sum_{\mathbf{p}} G\left(\mathbf{p}, \tilde{r}_{z}\right) .
\end{aligned}
$$

Последние величины связаны с поперечной и продольной восприимчивостями соответственно:

$$
\tilde{r}_{\perp}^{-1}=\int d \mathbf{r}\left\langle\sigma^{\prime \alpha}(\mathbf{r}) \sigma^{\prime \alpha}(\mathbf{0})\right\rangle, \quad \alpha=x, y, \quad \tilde{r}_{z}^{-1}=\int d \mathbf{r}\left\langle\sigma^{\prime z}(\mathbf{r}) \sigma^{\prime z}(\mathbf{0})\right\rangle .
$$

Отметим, что в этом случае справедливо соотношение

$$
\tilde{r}_{z}=\frac{\partial h}{\partial m_{z}} .
$$

С повышением температуры достигается критическое значение $T_{\mathrm{c}}$, выше которого исчезает $m_{\perp}$ и система переходит в парамагнитную фазу, известную как планарный парамагнетик [33] (см. рис. 5).

Вычитая (17) из (19), находим

$$
\tilde{r}_{\perp}=\frac{h}{m_{z}}+r_{x}-r_{z}+u m_{\perp}^{2}+\mathcal{O}(u) .
$$

Используя равенство $r_{x}-r_{z}=-3 g / 2-D^{2} / J_{z}$ и полагая $g<0$, что соответствует режиму “легкая ось", получаем, что в нулевом магнитном поле обратная поперечная восприимчивость

$$
\tilde{r}_{\perp}=\frac{3}{2}|g|-\frac{D^{2}}{J_{z}}+\mathcal{O}(u)
$$


остается конечной для изинговского режима $\left(|g|>2 D^{2} / 3 J_{z}\right)$ и расходится в гейзенберговском режиме $\left(|g|=2 D^{2} / 3 J_{z}\right)$. Это согласуется с фазовой диаграммой, полученной Клейном и Аарони из ренормгруппового анализа парамагнитной фазы [29], [30].

Пренебрегая в дальнейшем одноосной анизотропией (полагая $g=0$ ) и учитывая, что $m_{z}=h /\left(D^{2} / J_{z}\right)$, получаем из уравнения (23) соотношение

$$
\tilde{r}_{\perp}=u m_{\perp}^{2}+\mathcal{O}(u),
$$

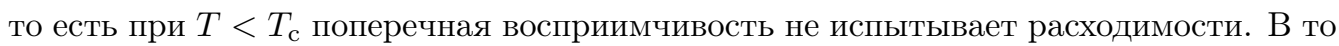
же время значение $\tilde{r}_{z}$ остается конечным даже выше критической температуры $T_{\mathrm{c}}$. Действительно, как следует из уравнения (22),

$$
\tilde{r}_{z}=\frac{h}{m_{z}}+u m_{z}^{2}
$$

Уравнения (17), (18) дают возможность получить температурную зависимость поперечной намагниченности в критической области. Следует учитывать, что при оценке сумм в (17), (18) необходимо вычитать из этих соотношений их выражения, вычисленные в критической точке. Соответствующие расчеты приводятся в приложении А.

Нужное равенство следует, например, из уравнения (18):

$0=r_{x}-r_{x \mathrm{c}}+\frac{u}{2} m_{\perp}^{2}+\frac{u}{2}(n+1) \sum_{\mathbf{p}}\left[G\left(\mathbf{p}, \tilde{r}_{\perp}\right)-G\left(\mathbf{p}, \tilde{r}_{\perp \mathrm{c}}\right)\right]+\frac{u}{2} \sum_{\mathbf{p}}\left[G\left(\mathbf{p}, \tilde{r}_{z}\right)-G\left(\mathbf{p}, \tilde{r}_{z \mathrm{c}}\right)\right]$.

Используя (А.3), получаем с помощью соотношений (25), (26), что

$$
0=r_{x}-r_{x \mathrm{c}}+\frac{u}{2} m_{\perp}^{2}+\mathcal{O}\left(u^{3 / 2}\right)
$$

и это обеспечивает закон температурного поведения поперечной намагниченности вида $m_{\perp} \sim \sqrt{T_{\mathrm{c}}-T}$.

Исследуем поведение системы в критической точке, когда внешнее магнитное поле мало. В этом случае возникает физическая ситуация, присущая ферромагнетику при фазовом переходе в парамагнитную фазу, когда поперечная компонента намагниченности обращается в нуль, в то время как продольная малая компонента намагниченности остается конечной.

Вычтем из уравнения (17) соответствующее выражение при $T=T_{\mathrm{c}}, h=m=0$, получим

$$
\frac{h}{m_{z}}=\frac{u}{2} m_{z}^{2}+\frac{u}{2}(n-1) \sum_{\mathbf{p}}\left[G\left(\mathbf{p}, \tilde{r}_{\perp}\right)-G(\mathbf{p}, 0)\right]+\frac{3}{2} u \sum_{\mathbf{p}}\left[G\left(\mathbf{p}, \tilde{r}_{z}\right)-G(\mathbf{p}, 0)\right] .
$$

Используя (А.4) и результат (В.3) для $u$, находим

$$
\frac{h}{m_{z}}=\frac{u}{2} m_{z}^{2}+\frac{\varepsilon(n-1)}{2(n+8)} \tilde{r}_{\perp} \ln \tilde{r}_{\perp}+\frac{3 \varepsilon}{2(n+8)} \tilde{r}_{z} \ln \tilde{r}_{z} .
$$

Из соотношений $(19),(20)$ имеем $\tilde{r}_{\perp} \approx u m_{z}^{2} / 2$ и $\tilde{r}_{z} \approx 3 u m_{z}^{2} / 2$. Подставляя эти выражения в уравнение (29), получаем

$$
\frac{h}{m_{z}} \propto \frac{u}{2} m_{z}^{2}\left(1+\varepsilon \ln m_{z}\right)
$$

что обеспечивает скейлинг $h \propto m_{z}^{3+\varepsilon}$, который предсказывает теория Вильсона [34]. 
3.3. Солитонная решетка. Рассмотрим фазу солитонной решетки, которая характеризуется магнитным упорядочением $\mathbf{S}(z)=m_{0}(\cos \varphi(z), \sin \varphi(z), 0)$. В этом случае

$$
\varphi(z)=\pi+2 \operatorname{am}\left(\frac{2 K z}{L_{\mathrm{CSL}}}, \kappa\right),
$$

где $\operatorname{am}(\cdot)$ - эллиптическая функция амплитуды Якоби, $K$ - эллиптический интеграл первого рода, $L_{\mathrm{CSL}}$ - пространственный период солитонной решетки. Солитонная решетка возникает под действием внешнего магнитного поля, перпендикулярного оси $z, \mathbf{h}=h \mathbf{e}_{x}$. Параметр $\kappa$ определяется из условия

$$
\frac{\sqrt{h}}{\kappa}=\frac{\pi Q_{0}}{4 E}
$$

Фурье-компоненты с $\mathbf{k}=\left(\mathbf{k}_{\perp}, k_{z}\right)$ заданы соотношением

$$
S_{\mathbf{k}}^{\alpha}=\delta_{\mathbf{k}_{\perp}, \mathbf{0}} \sum_{n=-\infty}^{+\infty} S_{n}^{\alpha} \delta\left(k_{z}-n G_{\mathrm{MKC}}\right), \quad \alpha=x, y,
$$

в котором

$$
S_{n}^{x}=\left\{\begin{array}{cl}
2 \pi m_{0}\left(\frac{2(K-E)}{\kappa^{2} K}-1\right), & n=0, \\
-2 \pi m_{0}\left(\frac{\pi}{\kappa K}\right)^{2} \frac{n}{\operatorname{sh}\left(\pi n K^{\prime} / K\right)}, & n \neq 0,
\end{array} \quad S_{n}^{y}=-2 i \pi m_{0}\left(\frac{\pi}{\kappa K}\right)^{2} \frac{n}{\operatorname{ch}\left(\pi n K^{\prime} / K\right)} .\right.
$$

Здесь, $G_{\mathrm{CSL}}=2 \pi / L_{\mathrm{CSL}}-$ волновой вектор модуляции солитонной решетки, $K^{\prime}-$ полный эллиптический интеграл первого рода от дополнительного модуля $\kappa^{\prime}=\sqrt{1-\kappa^{2}}$. Для получения этих результатов использованы ряды Фурье для эллиптических функций:

$$
\begin{aligned}
\operatorname{sn}^{2}(\bar{z}, \kappa) & =\frac{K-E}{\kappa^{2} K}-\left(\frac{\pi}{\kappa K}\right)^{2} \sum_{n=1}^{\infty} \frac{n \cos (\pi n \bar{z} / K)}{\operatorname{sh}\left(\pi n K^{\prime} / K\right)}, \\
\operatorname{sn}(\bar{z}, \kappa) \operatorname{cn}(\bar{z}, \kappa) & =\left(\frac{\pi}{\kappa K}\right)^{2} \sum_{n=1}^{\infty} \frac{n \sin (\pi n \bar{z} / K)}{\operatorname{ch}\left(\pi n K^{\prime} / K\right)}
\end{aligned}
$$

где $\bar{z}=2 K z / L_{\mathrm{MKC}}$.

Вычисляя спиновые переменные (7), имеем

$$
\sigma_{\mathbf{k}}^{\alpha}=\sum_{n=-\infty}^{+\infty} \sum_{p= \pm 1}\left(\delta_{\alpha, x}+p \delta_{\alpha, y}\right) \sigma_{n}^{\alpha} \delta\left(\mathbf{k}-p \mathbf{Q}_{0}-p n G_{\mathrm{MKC}} \mathbf{e}_{z}\right) \delta\left(\mathbf{k}_{\perp}\right),
$$

где

$$
\sigma_{n}^{x}=\pi m_{0}\left(\frac{2(K-E)}{\kappa^{2} K}-1\right) \delta_{n, 0}-2 \pi m_{0}\left(\frac{\pi}{\kappa K}\right)^{2} \frac{n \exp \left(-\pi n K^{\prime} / K\right)}{\operatorname{sh}\left(2 \pi n K^{\prime} / K\right)} \delta_{n \neq 0}
$$

и $\sigma_{n}^{y}=-i \sigma_{n}^{x}$. Для малого магнитного поля, когда $\kappa \ll 1$ и $G_{\mathrm{Mkc}} \approx Q_{0}$, ведущие слагаемые в разложениях (34) определяются выражениями

$$
\sigma_{\mathbf{k}}^{x} \approx 2 \sigma_{-1}^{x} \delta_{\mathbf{k}, \mathbf{0}}+\left(\sigma_{0}^{x}+\sigma_{-2}^{x}\right) \delta_{\mathbf{k}, \pm \mathbf{Q}_{0}}, \quad \sigma_{\mathbf{k}}^{y} \approx \pm \sigma_{0}^{y} \delta_{\mathbf{k}, \pm \mathbf{Q}_{0}} .
$$


С учетом равенств $\sigma_{-1}^{x}=-\pi m_{0}$ и $\sigma_{-2}^{x}+\sigma_{0}^{x}=0+\mathcal{O}\left(\kappa^{6}\right)$ имеем

$$
\begin{aligned}
& m_{\mathbf{k}}^{x} \approx-2 \pi m_{0} \delta_{\mathbf{k}, \mathbf{0}}, \\
& m_{\mathbf{k}}^{y} \approx-i \pi m_{0} \frac{\kappa^{2}}{8} \delta_{\mathbf{k}, \mathbf{Q}_{0}}+i \pi m_{0} \frac{\kappa^{2}}{8} \delta_{\mathbf{k},-\mathbf{Q}_{0}} .
\end{aligned}
$$

При выводе этих соотношений использованы разложения

$$
K=\frac{\pi}{2}\left(1+\frac{\kappa^{2}}{4}+\frac{9}{64} \kappa^{4}+\cdots\right), \quad E=\frac{\pi}{2}\left(1-\frac{\kappa^{2}}{4}-\frac{3}{64} \kappa^{4}+\cdots\right)
$$

и тот факт, что $\lim _{\kappa \rightarrow 0} K^{\prime}=\ln (4 / \kappa)$.

Внешнее поле, сопряженное $m_{\mathbf{k}}^{\alpha}$, обладает ненулевыми компонентами

$$
\tilde{h}_{-\mathbf{Q}_{0}}^{x}=\tilde{h}_{\mathbf{Q}_{0}}^{x}=\frac{h}{2}, \quad \tilde{h}_{-\mathbf{Q}_{0}}^{y}=-\tilde{h}_{\mathbf{Q}_{0}}^{y}=i \frac{h}{2} .
$$

Тогда уравнения состояния для $m_{\mathbf{0}}^{x}$ и $m_{ \pm \mathbf{Q}_{0}}^{y}$, полученные из $(15)$ с точностью $\mathcal{O}\left(\kappa^{4}\right)$, имеют соответственно вид

$$
\begin{aligned}
0 & =r_{x}+2 u \pi^{2} m_{0}^{2}+\frac{3 u}{2} \sum_{\mathbf{p}} G\left(\mathbf{p}, \tilde{r}_{x}\right)+\frac{u}{2} \sum_{\mathbf{p}} G\left(\mathbf{p}, \tilde{r}_{y}\right), \\
\frac{4 h}{\pi m_{0} \kappa^{2}} & =r_{y}+\Delta Q_{0}^{2}+2 u \pi^{2} m_{0}^{2}+\frac{3 u}{2} \sum_{\mathbf{p}} G\left(\mathbf{p}, \tilde{r}_{y}\right)+\frac{u}{2} \sum_{\mathbf{p}} G\left(\mathbf{p}, \tilde{r}_{x}\right),
\end{aligned}
$$

где

$$
\begin{aligned}
& \tilde{r}_{x}=r_{x}+6 u \pi^{2} m_{0}^{2}+\frac{3 u}{2} \sum_{\mathbf{p}} G\left(\mathbf{p}, \tilde{r}_{x}\right)+\frac{u}{2} \sum_{\mathbf{p}} G\left(\mathbf{p}, \tilde{r}_{y}\right), \\
& \tilde{r}_{y}=r_{y}+2 u \pi^{2} m_{0}^{2}+\frac{3 u}{2} \sum_{\mathbf{p}} G\left(\mathbf{p}, \tilde{r}_{y}\right)+\frac{u}{2} \sum_{\mathbf{p}} G\left(\mathbf{p}, \tilde{r}_{x}\right) .
\end{aligned}
$$

Уравнение (39) отражает независимость ферромагнитной компоненты (37) от внешнего магнитного поля. Это становится очевидным из разложения функции $\mathrm{am}(\cdot)$ в ряд Фурье, что дает

$$
\varphi(z)=\pi+G_{\mathrm{MKC}} z+\sum_{n=1}^{\infty} \frac{\sin \left(n G_{\mathrm{MKC}} z\right)}{n \operatorname{ch}\left(\pi n K^{\prime} / K\right)} .
$$

При приближении к точке фазового перехода соизмеримая фаза-несоизмеримая фаза, $G_{\text {мкс }}$ стремится к нулю, что приводит к значению $\varphi(z)=\pi$, согласующемуся с выражением (37). Величина $\tilde{r}_{x}=4 u \pi^{2} m_{0}^{2}$ остается конечной в точке перехода.

Вычитая (42) из (40), получаем тождество Уорда

$$
\tilde{r}_{y}=Q_{0}^{2}\left(\frac{1}{\pi m_{0}}-\Delta\right)
$$

где использовано выражение (32) для солитонной решетки. Из него следует, что несоизмеримая фаза стабильна при условии $\Delta \pi m_{0}<1$ или

$$
\frac{J_{\perp}}{J_{z}}>\pi m_{0} .
$$


Очевидно, что для большей величины спина $m_{0}$ требуется бо́льшая “двумерность” системы.

Критическая точка, в которой возникает стабилизация волнового вектора соизмеримой фазы $\mathbf{K}_{0}$, соответствует фазовому переходу соизмеримая фаза-несоизмеримая фаза. Согласно имеющимся экспериментальным данным для соединения $\mathrm{CrNb}_{3} \mathrm{~S}_{6}$ [20] с увеличением температуры начальная несоизмеримая фаза сменяется соизмеримой фазой с $\mathbf{K}_{0}=0$, что соответствует ферромагнитному упорядочению слоев. Более детально, при $T_{1 \mathrm{c}}=117 \mathrm{~K}$ поперечная магнитная восприимчивость, измеренная в слабых полях, приложенных перпендикулярно геликоидальной $c$-оси, имеет острый пик, что свидетельствует о возникновении геликоидального магнитного порядка при температурах ниже $T_{1 \mathrm{c}}$. Вторая критическая температура $T_{2 \mathrm{c}}=129 \mathrm{~K}$ связана с кроссовером из ферромагнитной в парамагнитую фазы, когда с ростом температуры разрушается трехмерный магнитный порядок.

Вычитая из уравнения (40) это же соотношение при $T_{1 \mathrm{c}}$, когда $Q_{0}$ обращается в нуль, находим

$$
Q_{0}^{2}\left(\frac{1}{\pi m_{0}}-\Delta\right)=r_{y}-r_{y 1}+\mathcal{O}(u)
$$

Это дает результат $Q_{0} \propto\left(T_{1 \mathrm{c}}-T\right)^{1 / 2}$, для получения которого использовано определение $r_{y} \sim \rho=a\left(T_{2 \mathrm{c}}-T\right)$.

3.4. Фазовый переход ферромагнитная фаза-парамагнитная фаза. Для описания кроссовера из соизмеримой ферромагнитной фазы в парамагнитную фазу вблизи критической точки $T_{2 \text { c }}$ в присутствии магнитного поля используем выражения $\mathbf{h}=h \mathbf{e}_{x}$ и $\mathbf{m}=m_{0} \mathbf{e}_{x}$. В этом случае $\mathbf{Q}_{0}=0$, что дает $\tilde{h}_{\mathbf{k}}^{x}=\tilde{h}_{\mathbf{0}}^{x}=h \delta_{\mathbf{k}, \mathbf{0}} \mathbf{e}_{x}$ и определяет уравнение состояния

$$
\frac{h}{m_{0}}=r_{x}+\frac{u}{2} m_{0}^{2}+\frac{u}{2}(n-1) \sum_{\mathbf{p}} G\left(\mathbf{p}, \tilde{r}_{\perp}\right)+\frac{3}{2} u \sum_{\mathbf{p}} G\left(\mathbf{p}, \tilde{r}_{x}\right)
$$

в котором

$$
\begin{aligned}
& \tilde{r}_{\perp}=r_{\perp}+\frac{u}{2} m_{0}^{2}+\frac{u}{2}(n+1) \sum_{\mathbf{p}} G\left(\mathbf{p}, \tilde{r}_{\perp}\right)+\frac{u}{2} \sum_{\mathbf{p}} G\left(\mathbf{p}, \tilde{r}_{x}\right), \\
& \tilde{r}_{x}=r_{x}+\frac{3}{2} u m_{0}^{2}+\frac{u}{2}(n-1) \sum_{\mathbf{p}} G\left(\mathbf{p}, \tilde{r}_{\perp}\right)+\frac{3}{2} u \sum_{\mathbf{p}} G\left(\mathbf{p}, \tilde{r}_{x}\right) .
\end{aligned}
$$

Дальнейший анализ воспроизводит результаты работ [31], [35]. Вычитая из уравне-

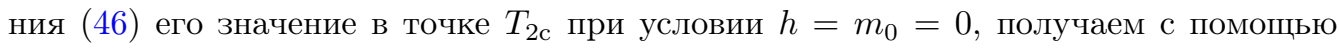
соотношений (А.4), (В.3) уравнение

$$
\frac{h}{m_{0}}=t+\frac{u}{2} m_{0}^{2}+\frac{\varepsilon}{2(n+8)}\left\{(n-1) \tilde{r}_{\perp} \ln \tilde{r}_{\perp}+3 \tilde{r}_{x} \ln \tilde{r}_{x}\right\},
$$

где $t=r_{x}-r_{x c}$ и $r_{x c}=r_{x}\left(T_{2 \mathrm{c}}\right)$. Чтобы получить критическое поведение с точностью $\mathcal{O}\left(\varepsilon^{2}\right)$, можно ограничиться соотношениями $\tilde{r}_{\perp}=t+u m_{0}^{2} / 2$ и $\tilde{r}_{x}=t+3 u m_{0}^{2} / 2$, где учтено, что в критической точке $\tilde{r}_{\perp}=\tilde{r}_{x}=0$. 
При $T=T_{2 \mathrm{c}}$, когда $t=0$, легко находим, что $h \propto m_{0}^{3+\varepsilon}$. При $T<T_{2 \mathrm{c}}$ имеем $\tilde{r}_{\perp}=0$, если $h=0$, что приводит к стандартному значению $\varepsilon$-разложения $m \propto(-t)^{\beta}$, в котором

$$
\beta=\frac{1}{2}-\frac{3 \varepsilon}{2(n+8)}
$$

это согласуется с критическими индексами трехмерной модели Гейзенберга.

\section{4. ЗАКЛЮЧЕНИЕ}

Перечислим основные результаты нашего исследования. Проанализировано критическое поведение модели одноосного кирального гелимагнетика с анизотропным обменным взаимодействием, которая представляет собой прототип реального соединения $\mathrm{CrNb}_{3} \mathrm{~S}_{6}$. С помощью формализма уравнения состояния вблизи критической точки рассмотрены фазы, возникающие в слабом внешнем магнитном поле, а именно коническая фаза, которая появляется под действием магнитного поля, направленного вдоль геликоидальной оси, и фаза солитонной решетки, которая стабилизируется полем, приложенным перпендикулярно геликоидальной оси.

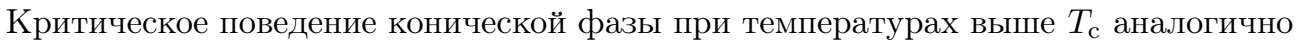
поведению планарного парамагнетика в присутствии поля, направленного перпендикулярно плоскости. Приближаясь к критической точке снизу, поперечная составляющая обращается в нуль по закону $m_{\perp} \sim\left(T_{\mathrm{c}}-T\right)^{1 / 2}$, тогда как продольная

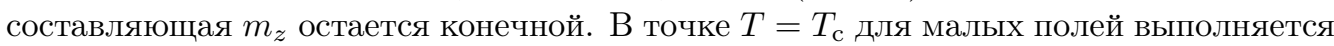
скейлинг $h \propto m_{z}^{3+\varepsilon}$, который согласуется с теорией Вильсона.

В случае приложенного перпендикулярного поля происходит следующая последовательность фазовых переходов при повышении температуры. Низкотемпературный несоизмеримый порядок заменяется ферромагнитной соизмеримой фазой при $T_{1 \mathrm{c}}$, а затем следует кроссовер к парамагнитному состоянию вблизи критической температуры $T_{2 \mathrm{c}}$. В режиме слабого поля при приближении к критической точке "соизмеримая фаза-несоизмеримая фаза" волновой вектор солитонной решетки изменяется по закону $Q_{0} \sim\left(T_{1 \mathrm{c}}-T\right)^{1 / 2}$. Фурье-разложение решения солитонной решетки показывает, что гармоники, кратные $\mathbf{Q}_{0}$, исчезают; при этом вблизи данного фазового перехода сохраняется только ферромагнитная составляющая. Это соответствует переходу от мульти- $Q$ фазы (солитонная решетка) к однокомпомонентной $Q$-фазе (вынужденный ферромагнетик), что проявляет себя как сингулярность кривой намагничивания $\mathrm{CrNb}_{3} \mathrm{~S}_{6}$ ниже температуры магнитного упорядочения.

Кроссовер к парамагнитному поведению характеризуется критическими показателями трехмерной модели Гейзенберга, и это согласуется с экспериментальным анализом скейлинга вблизи температуры Кюри в соединении $\mathrm{CrNb}_{3} \mathrm{~S}_{6}$. Данный вывод подтверждается недавним численным исследованием трехмерной классической модели кирального гелимагнетика с помощью метода Монте-Карло в работе [28], где было показано, что в отсутствии внешнего магнитного поля система испытывает непрерывный фазовый переход с критическими индексами трехмерной $X Y$-модели (что отвечает случаю $n=2$ нашего анализа). Таким образом, исследование критического поведения магнитоупорядоченных фаз моноаксиального кирального гелимагнетика подтвеждает главный результат ренормгруппового анализа парамагнитной фазы о том, что взаимодействие ДМ не приводит к каким-либо дополнительным 
кроссоверным индексам. Отметим, что вопрос о природе фазовых переходов в конечных магнитных полях остается открытым и нельзя исключать возникновение фазовых переходов первого рода.

На основе тождества Уорда получено условие устойчивости солитонной решетки в терминах отношения внутрислойного и межслойного взаимодействий, из которого следует, что сильный обмен $J_{z}$ может разрушать это состояние. Этот критерий разрешает давнюю проблему, почему правомерно рассматривать слоистое соединение $\mathrm{CrNb}_{3} \mathrm{~S}_{6}$ с использованием квазиклассической одномерной спиновой модели [9]. В пределе сильного взаимодействия $J_{\perp}$ динамические флуктуации внутри слоя оказываются полностью замороженными и устанавливается жесткое внутриплоскостное ферромагнитное упорядочение. Это позволяет рассматривать данное соединение как одномерную квазиклассическую систему. Недавнее моделирование методом Монте-Карло [36] дает оценки $J_{z}=8.0 \mathrm{~K}$ и $J_{\perp}=70 \mathrm{~K}$, что полностью поддерживает это заключение.

\section{Вычисление интегралов}

ПРИЛОЖЕНИЕ А

Для вывода уравнений состояния необходимо определить величину

$$
\begin{aligned}
I_{1} & =\sum_{\mathbf{p}}\left(G\left(\mathbf{p}, \tilde{r}_{\alpha}\right)-G(\mathbf{p}, 0)\right)=\sum_{\mathbf{p}}\left(\frac{1}{\tilde{r}_{\alpha}+p_{\perp}^{2}+\Delta p_{z}^{2}}-\frac{1}{p_{\perp}^{2}+\Delta p_{z}^{2}}\right)= \\
& =-\tilde{r}_{\alpha} \int \frac{d^{3} p}{(2 \pi)^{3}} \frac{1}{\left(\tilde{r}_{\alpha}+p_{\perp}^{2}+\Delta p_{z}^{2}\right)\left(p_{\perp}^{2}+\Delta p_{z}^{2}\right)} .
\end{aligned}
$$

Используя правило Фейнмана

$$
\frac{1}{A B}=\int_{0}^{1} d x \frac{1}{[A x+B(1-x)]^{2}}
$$

получаем

$$
\begin{aligned}
I_{1} & =-\tilde{r}_{\alpha} \int \frac{d^{3} p}{(2 \pi)^{3}} \int_{0}^{1} d x \frac{1}{\left[\tilde{r}_{\alpha} x+p_{\perp}^{2}+\Delta p_{z}^{2}\right]^{2}}= \\
& =-\frac{\tilde{r}_{\alpha}}{4 \pi^{2}} \int_{0}^{1} d x \int_{0}^{\infty} p_{\perp} d p_{\perp} \int_{-\infty}^{+\infty} d p_{z} \frac{1}{\left[\tilde{r}_{\alpha} x+p_{\perp}^{2}+\Delta p_{z}^{2}\right]^{2}}
\end{aligned}
$$

Прямой расчет приводит к результату

$$
\sum_{\mathbf{p}}\left(G\left(\mathbf{p}, \tilde{r}_{\alpha}\right)-G(\mathbf{p}, 0)\right)=-\frac{1}{4 \pi} \sqrt{\frac{\tilde{r}_{\alpha}}{\Delta}}
$$

Чтобы использовать технику в-разложения для анализа переходов из парамагнитной фазы, расчет суммы $I_{1}$ выполняется для размерности $d=4-\varepsilon$. Поскольку для упорядоченных фаз мы ограничиваемся расчетом критических индексов до первого порядка по $\varepsilon$, достаточно вычислить сумму при $d=4$. Таким образом,

$$
I_{1}=\sum_{\mathbf{p}}\left(G\left(\mathbf{p}, \tilde{r}_{\alpha}\right)-G(\mathbf{p}, 0)\right)=\int \frac{d^{3} p_{\perp}}{(2 \pi)^{3}} \int_{-\Lambda_{z}}^{\Lambda_{z}} \frac{d p_{z}}{(2 \pi)}\left[\frac{1}{\tilde{r}_{\alpha}+p_{\perp}^{2}+\Delta p_{z}^{2}}-\frac{1}{p_{\perp}^{2}+\Delta p_{z}^{2}}\right] .
$$




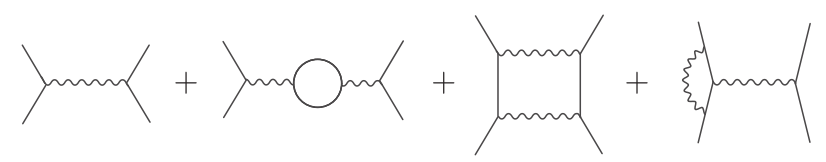

Рис. 6. Диаграммы для вычисления $\Gamma_{4}$.

Предполагая, что $p_{\perp} \leqslant \Lambda<\Lambda_{z} \sqrt{\Delta}$, имеем

$$
I_{1} \approx \frac{1}{4 \pi^{2} \sqrt{\Delta}} \int_{0}^{\Lambda} p_{\perp}^{2} d p_{\perp}\left[\frac{1}{\sqrt{\tilde{r}_{\alpha}+p_{\perp}^{2}}}-\frac{1}{p_{\perp}}\right]=\frac{1}{8 \pi^{2} \sqrt{\Delta}}\left\{-\tilde{r}_{\alpha} \ln (2 \Lambda)+\frac{1}{2} \tilde{r}_{\alpha} \ln \tilde{r}_{\alpha}\right\} .
$$

Вблизи критической точки $\tilde{r}_{\alpha} \ll 1$ получаем окончательный результат:

$$
\sum_{\mathbf{p}}\left(G\left(\mathbf{p}, \tilde{r}_{\alpha}\right)-G(\mathbf{p}, 0)\right) \approx \frac{1}{16 \pi^{2} \sqrt{\Delta}} \tilde{r}_{\alpha} \ln \tilde{r}_{\alpha}
$$

ПРИЛОЖЕНИЕ В

\section{Вычисление константы взаимодействия}

Для расчета критического поведения в окрестности фазового перехода в парамагнитную фазу, где все $\tilde{r}_{\alpha}$ малы, нужно взять значение $u$ пропорциональным $\varepsilon$. Для этой цели используется четырехточечная спиновая корреляционная функция [34], которая масштабируется в первом порядке по $\varepsilon$ следующим образом:

$$
\Gamma_{4}\left(\tilde{r}_{\alpha}\right) \sim 1+\frac{\varepsilon}{2} \ln \tilde{r}_{\alpha}+\mathcal{O}\left(\varepsilon^{2}\right) .
$$

С другой стороны, функцию $\Gamma_{4}\left(\tilde{r}_{\alpha}\right)$ можно рассчитать с помощью диаграммного ряда. Диаграммы первого и второго порядка по взаимодействию $u$ показаны на рис. 6. С их помощью получаем соотношение

$$
\Gamma_{4}\left(\tilde{r}_{\alpha}\right)=u-u^{2}\left(\frac{n}{2}+4\right) \int \frac{d^{d} p}{(2 \pi)^{d}} G^{2}\left(\mathbf{p}, \tilde{r}_{\alpha}\right)=u-u^{2}\left(\frac{n}{2}+4\right) \int \frac{d^{d} p}{(2 \pi)^{d}} \frac{1}{\left(\tilde{r}_{\alpha}+p_{\perp}^{2}+\Delta p_{z}^{2}\right)^{2}} .
$$

Прямой расчет дает

$$
\Gamma_{4}\left(\tilde{r}_{\alpha}\right) \propto u\left[1+u\left(\frac{n}{2}+4\right) \frac{1}{16 \pi^{2} \sqrt{\Delta}} \ln \tilde{r}_{\alpha}\right] .
$$

Непосредственное сравнение с (В.1) приводит к выражению

$$
u=\frac{16 \pi^{2}}{n+8} \varepsilon \sqrt{\Delta} .
$$

Благодарности. Авторы выражают признательность Д. Чернышеву, Х. М. Рённо, С. В. Григорьеву и Ю. Като за дискуссии. 


\section{Список литературы}

[1] Y. Ishikawa, K. Tajima, D. Bloch, M. Roth, "Helical spin structure in manganese silicide MnSi", Solid State Commun., 19:6 (1976), 525-528.

[2] S. Mühlbauer, B. Binz, F. Jonietz, C. Pfleiderer, A. Rosch, A. Neuebauer, R. Georgii, P. Böni, "Skyrmion lattice in a chiral magnet", Science, 323:5916 (2009), 915-919.

[3] X. Z. Yu, Y. Onose, N. Kanazawa, J. H. Park, J. H. Han, Y. Matsui, N. Nagaosa, Y. Tokura, "Real-space observation of a two-dimensional skyrmion crystal", Nature, 465:7300 (2010), 901-904.

[4] W. Münzer, A. Neubauer, T. Adams, S. Mühlbauer, C. Franz, F. Jonietz, R. Georgii, P. Böni, B. Pedersen, M. Schmidt, A. Rosch, C. Pfleiderer, "Skyrmion lattice in the doped semiconductor $\mathrm{Fe}_{1-x} \mathrm{Co}_{x} \mathrm{Si}$ ", Phys. Rev. B, 81:4 (2010), 041203, 4 pp.

[5] B. Lebech, J. Bernhard, T. Freltoft, "Magnetic structures of cubic FeGe studied by smallangle neutron scattering", J. Phys.: Condens. Matter, 1:35 (1989), 6105-6122.

[6] X. Z. Yu, N. Kanazawa, Y. Onose, K. Kimoto, W. Z. Zhang, S. Ishiwata, Y. Matsui, Y. Tokura, "Near room-temperature formation of a skyrmion crystal in thin-films of the helimagnet FeGe", Nature Mater., 10:2 (2011), 106-109.

[7] K. Kohn, "A new ferrimagnet $\mathrm{Cu}_{2} \mathrm{SeO}_{4}$ ", J. Phys. Soc. Japan, 42:6 (1977), 2065-2066.

[8] S. Seki, X.Z. Yu, S. Ishiwata, Y. Tokura, "Observation of skyrmions in a multiferroic material", Science, 336:6078 (2012), 198-201.

[9] T. Moriya, T. Miyadai, "Evidence for the helical spin structure due to antisymmetric exchange interaction in $\mathrm{Cr}_{13} \mathrm{NbS}_{2}$ ", Solid State Commun., 42:3 (1982), 209-212.

[10] T. Miyadai, K. Kikuchi, H. Kondo, S. Sakka, M. Arai, Y. Ishikawa, "Magnetic properties of $\mathrm{Cr}_{13} \mathrm{NbS}_{2}$ ", J. Phys. Soc. Japan, 52:4 (1983), 1394-1401.

[11] U. K. Rößler, A. N. Bogdanov, C. Pfleiderer, "Spontaneous skyrmion ground states in magnetic metals", Nature, 442:7104 (2006), 797-801.

[12] N. Nagaosa, Y. Tokura, "Topological properties and dynamics of magnetic skyrmions", Nature Nanotech., 8:12 (2013), 899-911.

[13] И.Е. Дзялошинский, “Теория геликоидальных структур в антиферромагнетиках. I. Неметаллы”, ЖЭЭТФ, 46:4 (1964), 1420-1437; “Теория геликоидальных структур в антиферромагнетиках. ІІ. Металлы", ЖЭТФ, 47:1 (1965), 336-348.

[14] Ю.А. Изюмов, "Модулированные, или длиннопериодические, магнитные структуры кристаллов", УФН, 144:3 (1984), 439-474.

[15] Y. Togawa, T. Koyama, K. Takayanagi, S. Mori, Y. Kousaka, J. Akimitsu, S. Nishihara, K. Inoue, A. S. Ovchinnikov, J. Kishine, "Chiral magnetic soliton lattice on a chiral helimagnet", Phys. Rev. Lett., 108:10 (2012), 107202, 5 pp.

[16] J. Kishine, A. S. Ovchinnikov, "Theory of monoaxial chiral helimagnet", Solid State Physics, 66, eds. R. E. Camley, R. L. Stamps, Academic Press, New York, 2015, 1-130.

[17] I. Dzyaloshinskii, "A thermodynamic theory of 'weak' ferromagnetism of antiferromagnetics", J. Phys. Chem. Solids, 4:4 (1958), 241-255.

[18] T. Moriya, "Anisotropic superexchange interaction and weak ferromagnetism", Phys. Rev., 120:1 (1960), 91-98.

[19] Y. Kousaka, Y. Nakao, J. Kishine, M. Akita, K. Inoue, J. Akimitsu, "Chiral helimagnetism in $\mathrm{T}_{1 / 3} \mathrm{NbS}_{2}(\mathrm{~T}=\mathrm{Cr}$ and $\mathrm{Mn})$ ", Nucl. Instrum. Methods Phys. Res. A, 600:1 (2009), 250-253.

[20] N. J. Ghimire, M. A. McGuire, D. S. Parker, B. Sipos, S. Tang, J.-Q. Yan, B. C. Sales, D. Mandrus, "Magnetic phase transition in single crystals of the chiral helimagnet $\mathrm{Cr}_{1 / 3} \mathrm{NbS}_{2} "$, Phys. Rev. B, 87:10 (2013), 104403.

[21] S. V. Grigoriev, S. V. Maleyev, A. I. Okorokov, Yu. O. Chetverikov, H. Eckerlebe, "Field-induced reorientation of the spin helix in MnSi near $T_{\mathrm{c}}$ ", Phys. Rev. B, 73:22 (2006), 224440, 9 pp. 
[22] S. V. Grigoriev, V. A. Dyadkin, D. Menzel, J. Schoenes, Yu. O. Chetverikov, A. I. Okorokov, H. Eckerlebe, S. V. Maleyev, "Magnetic structure of $\mathrm{Fe}_{1-x} \mathrm{Co}_{x} \mathrm{Si}$ in a magnetic field studied via small-angle polarized neutron diffraction", Phys. Rev. B, 76:22 (2007), 224424, 10 pp.

[23] C. Pappas, E. Lelièvre-Berna, P. Falus, P. M. Bentley, E. Moskvin, S. Grigoriev, P. Fouquet, B. Farago, "Chiral paramagnetic skyrmion-like phase in MnSi", Phys. Rev. Lett., 102:19 (2009), 197202, 4 pp.

[24] С. А. Бразовский, "Фазовый переход изотропной системы в неоднородное состояние", ЖЭТФ, 68:1 (1975), 175-185.

[25] F. B. Mushenok, "Homogeneous and Goldstone modes of spin excitations in $\mathrm{Cr}_{1 / 3} \mathrm{NbS}_{2}$ helimagnet", Eur. Phys. J. B, 86:8 (2013), 342, 4 pp.

[26] V. Dyadkin, F. Mushenok, A. Bosak, D. Menzel, S. Grigoriev, P. Pattison, D. Chernyshov, "Structural disorder versus chiral magnetism in $\mathrm{Cr}_{1 / 3} \mathrm{NbS}_{2}$ ", Phys. Rev. B, 91:18 (2015), 184205, 7 pp.

[27] I. Živković, J. S. White, H. M. Rønnow, K. Prša, H. Berger, "Critical scaling in the cubic helimagnet $\mathrm{Cu}_{2} \mathrm{OSeO}_{3}$ ", Phys. Rev. B, 89:6 (2014), 060401, 5 pp.

[28] Y. Nishikawa, K. Hukushima, "Phase transitions and ordering structures of a model of chiral helimagnet in three dimensions", Phys. Rev. B, 94 (2016), 064428, 11 pp., arXiv: 1603.04200 .

[29] L. Klein, A. Aharony, "Crossover and multicriticality due to the Dzyaloshinsky-Moriya interaction", Phys. Rev. B, 44:2 (1991), 856-858.

[30] L. L. Liu, "Effect of antisymmetric interactions on critical phenomena: a system with helical ground state", Phys. Rev. Lett., 31:7 (1973), 459-462.

[31] E. Brézin, D. J. Wallace, K. G. Wilson, "Feynman-graph expansion for the equation of state near the critical point", Phys. Rev. B, 7:1 (1973), 232-239.

[32] S.-K. Ma, Modern Theory of Critical Phenomena, Benjamin, Reading, MA, 1976.

[33] B. I. Halperin, P. C. Hohenberg, "Scaling laws for dynamic critical phenomena", Phys. Rev., 177:2 (1969), 952-971.

[34] K. G. Wilson, J. Kogut, "The renormalization group and the $\epsilon$ expansion", Phys. Rep. C, 12:2 (1974), 75-199.

[35] A. Aharony, "Equation of state for cubic ferromagnets", Phys. Rev. B, 10:7 (1974), 3006-3009.

[36] M. Shinozaki, S. Hoshino, Y. Masaki, J. Kishine, Y. Kato, J. Phys. Soc. Japan, 85 (2016), 074710, 7 pp., arXiv: 1512.00235 . 